\title{
Superposition of two mesoscopically distinct quantum states: Coupling a Cooper-pair box to a large superconducting island
}

\author{
Florian Marquardt* and C. Bruder ${ }^{\dagger}$ \\ Departement Physik und Astronomie, Universität Basel, Klingelbergstrasse 82, CH-4056 Basel, Switzerland
}

(Received 5 July 2000; published 11 January 2001)

\begin{abstract}
We consider a system of two superconducting islands, each of which is coupled to a bulk superconductor via Josephson tunneling. One of the islands represents a "Cooper-pair box," i.e., it is an effective two-level system. The other island has a smaller charging energy and approximates a harmonic oscillator. A capacitive interaction between the islands results in a dependence of the oscillator frequency on the quantum state of the box. Placing the latter in a coherent superposition of its eigenstates and exciting coherent oscillations in the large island will lead to a phase shift of these oscillations depending on the box quantum state, thereby producing a coherent superposition of two "mesoscopically distinct" quantum states in the large island.
\end{abstract}

DOI: 10.1103/PhysRevB.63.054514

PACS number(s): 74.50.+r, 73.23.Hk, 03.67.Lx

\section{INTRODUCTION}

Recent experiments by Nakamura, Pashkin, and Tsai. ${ }^{1}$ have demonstrated the coherent quantum dynamics of a socalled "Cooper-pair box," consisting of a small capacitance superconducting island coupled to a bulk superconductor via Josephson tunneling. Due to the small capacitance, there can be only zero or one excess Cooper pair on the island, which leads to an effective two-level description of the system (at appropriate values of the external bias voltage). The possible use of these two-level systems as building blocks of a quantum computer had been suggested already prior to Nakamura's work (see Refs. 2 and 3). However, it is clear that realizing such a quantum computer even with a modest number of qubits will prove exceedingly difficult and progress will be made only in small steps. A first step in this direction will consist in the coupling of two such Cooper-pair boxes.

Here, we consider theoretically another possibility: the capacitive coupling to a larger superconducting island that has a comparatively small charging energy, see Fig. 1. Its dynamics will involve more charge states and would approach that of a harmonic oscillator in the limit $E_{J} / E_{C} \rightarrow \infty$, where $E_{J}$ and $E_{C}$ denote the Josephson coupling energy and the charging energy of the large island, respectively. ${ }^{4}$ This suggests a rough analogy to the case of a two-level atom interacting with a single mode of the quantized electromagnetic field. Such a system has been explored experimentally in the field of cavity quantum electrodynamics. In particular, it has been used to entangle the two-level atom's state with a coherent state of the field harmonic oscillator, thereby creating a kind of "Schrödinger cat" state, in which the phase of the coherent oscillations depends on the quantum state of the two-level system (see Refs. 5). Therefore, the quantum superposition of two different microscopic states has been carried over to a superposition of two mesoscopically distinct states. This has also been considered as a model system for studying both the measurement process and decoherence by coupling the "mesoscopic" system to the external states of the environment (decay of the cavity mode). Although a kind of Schrödinger cat state has been proposed theoretically before in the context of a single Josephson junction, using quite a different mechanism (see Ref. 6), it did not feature the entanglement of a "microscopic" with a "mesoscopic" system.

The most important ingredient of such a system consists in a way of making the harmonic oscillator frequency dependent on the quantum state of the two-level system. Coming back to the superconducting island, this would mean a dependence of $E_{J}$ or $E_{C}$ on the state of a two-level system, which does not seem to be feasible at the moment, although replacing the single Josephson junction by a SQUID that is threaded by the flux of a "phase qubit" (proposed by Mooij et $a l^{7}{ }^{7}$ ) could make the value of the effective $E_{J}$ depend on the state of that qubit. In the present work, we will consider another possibility, suggested by the analogy to the two-level atom, which changes the cavity mode frequency by an offresonant (dispersive) coupling between the two. In the setting of the two capacitively coupled islands, this can be realized by detuning the oscillation frequency of the "large island" and the Josephson frequency of the Cooper-pair box. It is also necessary to operate the box at the degeneracy point, where the charging energies of the two states (with and without one excess Cooper pair) coincide and where the eigenstates are superpositions of the two charge states. Since

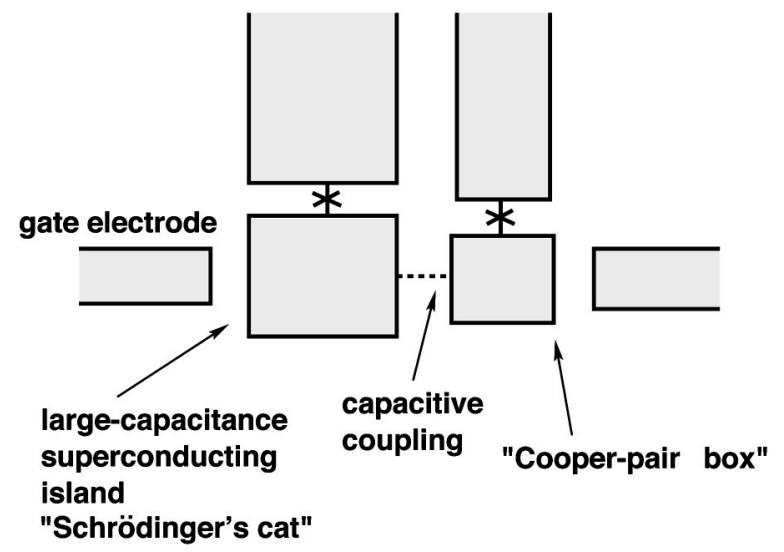

FIG. 1. Schematic diagram of the proposed experimental setup. The crosses denote Josephson junctions between each of the islands and the corresponding bulk superconductor. All other couplings are purely capacitive. The circuit diagram is shown in Fig. 3. 
the capacitive coupling is diagonal in the charge basis, it will be nondiagonal in the eigenbasis of the box, which will be essential for the proposed realization of a state-dependent frequency of the larger island.

\section{MODEL}

The Hamiltonian consists of the charging energies and Josephson coupling energies of both islands separately and an additional interaction term representing the purely capacitive coupling between the charges on both islands:

$$
\hat{H}=\hat{H}_{B}+\hat{H}_{O}+\hat{H}_{I},
$$

where the indices $B, O$, and $I$ refer to the Cooper-pair box, the large island ("'oscillator'") and the interaction, respectively. They are given by

$$
\begin{gathered}
\hat{H}_{B}=-E_{J B} \cos \left(\hat{\phi}_{B}\right)+E_{C B}\left(\hat{N}_{B}-N_{G B}\right)^{2}, \\
\hat{H}_{O}=-E_{J} \cos (\hat{\phi})+E_{C}\left(\hat{N}-N_{G}\right)^{2},
\end{gathered}
$$

and

$$
\hat{H}_{I}=E_{C C} \hat{N}\left(\hat{N}_{B}-\frac{1}{2}\right)
$$

Here, $\hat{N}$ and $\hat{N}_{B}$ are the operators corresponding to the number of excess Cooper pairs on the large and small islands, respectively, while $\hat{\phi}$ and $\hat{\phi}_{B}$ are the corresponding canonically conjugate phases. Gate voltages applied to both islands independently introduce offsets of $N_{G}$ and $N_{G B}$ for the charging energies. The Josephson coupling energies are given by $E_{J}, E_{J B}$, and the charging energy for a single Cooper pair is $E_{C}$ or $E_{C B}$. The strength of the interaction between the charges on both islands, resulting from the mutual capacitive coupling, is given by $E_{C C}$. The offset of $-1 / 2$ in the interaction energy $\hat{H}_{I}$ is introduced for convenience and could be compensated by a shift of the gate charge in $\hat{H}_{O}$. A calculation relating these parameters to the actual values of the capacitances and gate voltages is given in the Appendix.

The charging energy of the Cooper-pair box is assumed to be so large that it is permissible to neglect all charge states except for the two lowest lying ones. Initially, the Cooperpair box is prepared in an equal superposition of its two energy eigenstates by switching the gate offset $N_{G B}$ to the degeneracy point $N_{G B}=1 / 2$, starting at a value which is sufficiently far away from this point. The gate offset of the large island is first set to a value $N_{G} \gg 1$, which is then quickly switched to zero. We are interested in analyzing the subsequent quantum dynamics of the entire system, which will turn out to entangle the box states with two states of the large island that can be "mesoscopically distinct.",

\section{APPROXIMATION THROUGH A HARMONIC OSCILLATOR}

In the limit $E_{J} / E_{C} \gg 1$, the width $\delta N$ of the ground state of the large island is much larger than one. Therefore, we can treat the charge variable $\hat{N}$ as continuous and arrive at the model of a harmonic oscillator coupled to a two-level system ("Jaynes-Cummings model"' in quantum optics):

$$
\hat{H}_{O}=\sqrt{2 E_{J} E_{C}}\left[\frac{\left(\hat{q}-q_{G}\right)^{2}}{2}+\frac{\hat{p}^{2}}{2}+\frac{\omega_{B}}{2} \hat{\sigma}_{z}+\sqrt{2} g \hat{q} \hat{\sigma}_{x}\right],
$$

where $\hat{q}$ corresponds to the scaled charge variable (now taken to be continuous), $\hat{p}$ is its canonically conjugate momentum ( $\hbar$ has been set to 1 ), $\hat{\sigma}_{z}$ and $\hat{\sigma}_{x}$ act on the twolevel system whose Hamiltonian is diagonal in the $z$ basis with an energy difference of $E_{J B} \equiv \sqrt{2 E_{J} E_{C}} \omega_{B}$, and $g$ is a dimensionless measure of the coupling strength:

$$
\begin{gathered}
\hat{q} \equiv\left(\frac{E_{J}}{2 E_{C}}\right)^{-1 / 4} \hat{N}, \\
\omega_{B} \equiv \frac{E_{J B}}{\sqrt{2 E_{J} E_{C}}},
\end{gathered}
$$

$$
g \equiv \frac{E_{C C}}{4\left(2 E_{J} E_{C}^{3}\right)^{1 / 4}} .
$$

Note that the coupling term $\sim \hat{\sigma}_{x}$ between the charges on both islands is nondiagonal in the eigenbasis of the two-level system, since at the degeneracy point $N_{G B}=1 / 2$ the eigenstates are equally weighted superpositions of the two charge states.

In the context of Josephson junctions, the JaynesCummings model has been studied previously for the coupling to a single mode of the quantized electromagnetic field (see, e.g., Ref. 8) and to a superconducting resonator circuit. ${ }^{9}$

In the following, we will analyze the dimensionless Hamiltonian

$$
\hat{h}_{O} \equiv \frac{\hat{H}_{O}}{\sqrt{2 E_{J} E_{C}}},
$$

where the oscillator frequency is equal to 1 . Without the interaction, the eigenstates of $\hat{h}_{O}$ can be labeled $| \pm, n\rangle(n$ $=0,1,2, \ldots)$, where $n$ refers to the eigenstates of the harmonic oscillator and - and + stand for the ground state and excited state of the two-level system. The energies are given by

$$
\epsilon_{ \pm, n}=n+\frac{1}{2} \pm \frac{\omega_{B}}{2} .
$$

For a harmonic oscillator the procedure of switching the gate voltage (and thereby the offset $q_{G}$ of the harmonic oscillator potential) excites coherent oscillations of amplitude $q_{G}$ in the variable $\hat{q}$. The effect of the coupling to the two-levelsystem is to shift the frequency of the coherent oscillations by an amount that depends on the state of the two-level system. This can be analyzed most easily within the rotatingwave approximation to the Jaynes-Cummings model, where only the coupling between the almost degenerate states 
$|+, n\rangle$ and $|-, n+1\rangle$ is taken into account and the Hamiltonian is diagonalized within these two-dimensional subspaces. This is permissible as long as both the detuning $\delta \omega$ $\equiv 1-\omega_{B}$ between the oscillator and the two-level system and the coupling between them are much smaller than the frequency of the oscillator ( $\delta \omega \ll 1$ and $g \sqrt{n} \ll 1$ ). For the particular case of weak coupling considered here,

$$
g \sqrt{n} \ll \delta \omega,
$$

where the effective coupling strength is much smaller than the detuning $\delta \omega$, the most important effect on the quantum dynamics is due to the small shift in energy levels, given by

$$
\delta \epsilon_{-, n+1}=-\delta \epsilon_{+, n} \approx \frac{g^{2}(n+1)}{\delta \omega} .
$$

There is no shift for the state $|-, 0\rangle$ in this approximation. In the following, we will only take into account the energy shift, which leads to a slow change of the phase over the course of many oscillations. We will neglect the small change in the eigenstates, which is of order $g \sqrt{n} \delta \omega^{-1}$. It is unimportant for the present discussion, as it does not lead to such a phase shift growing in time.

An initial product state $\Psi_{ \pm}(0)=| \pm\rangle \otimes|\alpha\rangle$ consisting of one of the two eigenstates $| \pm\rangle$ of the two-level system and an oscillator coherent state

$$
|\alpha\rangle \equiv e^{-|\alpha|^{2} / 2} \sum_{n \geqslant 0} \frac{\alpha^{n}}{\sqrt{n !}}|n\rangle
$$

will evolve into

$$
\Psi_{ \pm}(t) \approx \exp \left[-i \varphi_{ \pm}(t)\right] \cdot| \pm\rangle \otimes\left|\alpha_{ \pm}(t)\right\rangle,
$$

where the global phase of the wave function is given by $\varphi_{ \pm}(t)$, whereas the time-evolved complex phase for the coherent oscillation is $\alpha_{ \pm}(t)$ :

$$
\begin{gathered}
\varphi_{+}(t)=\left(\frac{1+\omega_{B}}{2}-\frac{g^{2}}{\delta \omega}\right) t, \\
\varphi_{-}(t)=\frac{1-\omega_{B}}{2} t, \\
\alpha_{ \pm}(t)=\alpha \exp \left[-i\left(1 \mp \frac{g^{2}}{\delta \omega}\right) t\right] .
\end{gathered}
$$

Therefore, a phase difference with respect to the phase of the coherent oscillation develops between the two states and it is given by

$$
2 \frac{g^{2}}{\delta \omega} t
$$

Starting the system in a state

$$
\Psi(0)=\frac{1}{\sqrt{2}}(|+\rangle+|-\rangle) \otimes|\alpha\rangle
$$

will lead, after a time on the order of $\delta \omega / g^{2}$, to a superposition of two different coherent states of the harmonic oscillator. They will be "mesoscopically distinct," provided the amplitude of the coherent oscillations is larger than the width of the wave packet, i.e., the width of the ground state (which is $\approx 1$ in these dimensionless variables). These states are entangled with the states of the two-level system. In this way, a "Schrödinger cat state" has been produced.

In the following, we investigate numerically how this scenario changes when we go back to the original system of a superconducting island with a large but finite ratio $E_{J} / E_{C}$, instead of the ideal harmonic oscillator considered up to now in the rotating-wave approximation.

\section{NUMERICAL SIMULATION}

We have studied the effects of deviations from the ideal harmonic oscillator case by direct numerical simulation of the quantum dynamics resulting from the original Hamiltonian $\hat{H}_{O}$. These deviations are due to the fact that $\hat{N}$ is an operator with discrete eigenvalues in contrast to the continuous spatial coordinate $\hat{q}$. Viewed differently, the approximation of a harmonic oscillator is only valid in the limit of $E_{J} / E_{C} \rightarrow \infty$ when one can replace the $\cos \hat{\phi}$ term by the parabolic potential $1-\hat{\phi}^{2} / 2$.

The results shown in Fig. 2 have been obtained for the parameters $E_{J} / E_{C}=50, E_{J B} / E_{C}=9, E_{C C} / E_{C}=0.1$, and $E_{C B} / E_{C}=100$, so that the relative detuning between the frequency of the harmonic oscillator and the two-level system is

$$
\delta \omega=1-\frac{E_{J B}}{\sqrt{2 E_{J} E_{C}}}=0.1,
$$

whereas the dimensionless coupling strength is

$$
g=\frac{E_{C C}}{4\left(2 E_{J} E_{C}^{3}\right)^{1 / 4}} \approx 7.9 \times 10^{-3} .
$$

The initial gate charge of the oscillator was set to $N_{G}=2$ in this simulation. Note that this corresponds to about $1 / 3$ of the ground state's full width at half maximum (FWHM), so that this simulation is not yet in the parameter regime where the oscillator states become completely orthogonal after a while. (An increase in $N_{G}$ would lead to more pronounced squeezing, as described below.)

It is evident from Fig. 2 that the deviations from the ideal model result in a kind of squeezing for the amplitude of the oscillations and fluctuations in the time evolution of the phase difference.

\section{POSSIBLE EXPERIMENTAL REALIZATION}

Here we will first describe a possible experimental procedure to realize the "Schrödinger cat" states. At the end of this section, the various parameters and possible restrictions on them are discussed.

Initially, both the "box" and the harmonic oscillator have to be prepared in their ground states, which depend on the gate voltages applied to the two islands. The gate voltage of 

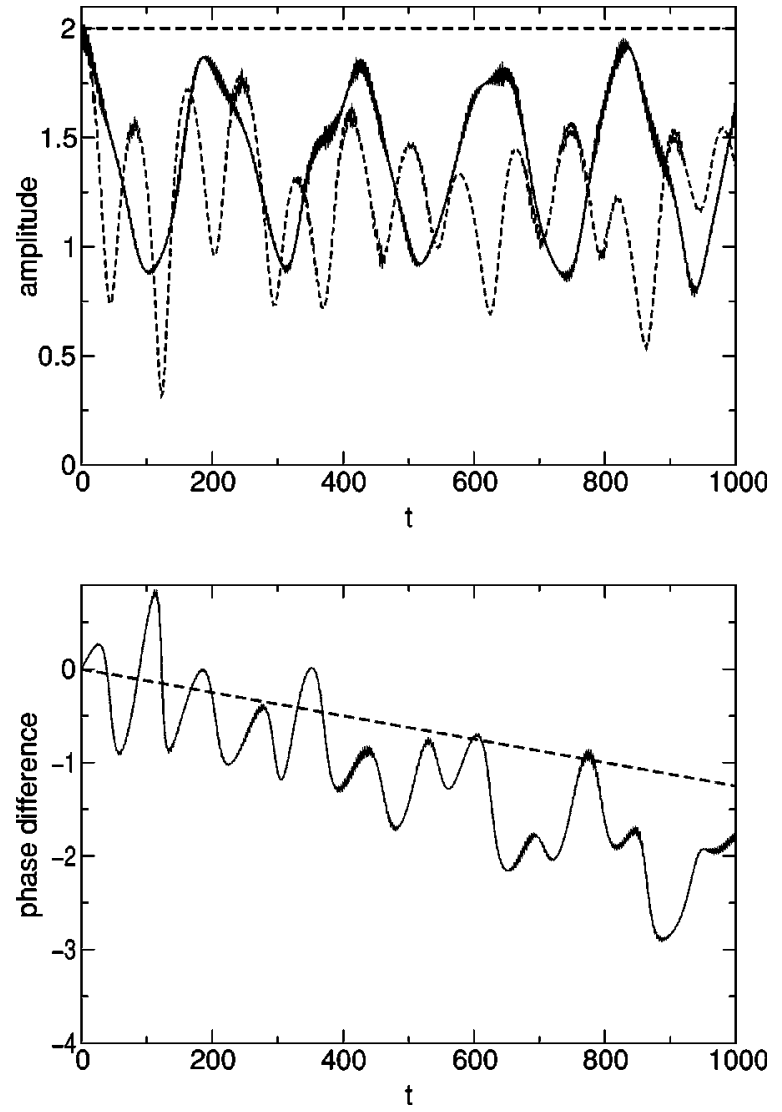

FIG. 2. Results of the numerical simulation for realistic parameters: In the upper figure, the envelope of the oscillations in the expectation value $\langle\hat{N}\rangle$ of the charge number in the large island is shown. This expectation value was calculated with respect to the two energy eigenstates $|+\rangle$ and $|-\rangle$ of the Cooper-pair box, resulting in the solid and dashed lines. In the ideal case discussed in the text, these envelopes should be constant and equal to the initial value of 2. The lower figure displays the time evolution of the difference between the phases of the two oscillations, where the straight line gives the theoretical expectation for the ideal harmonic oscillator (see text). Note that the time $t$ is given in dimensionless units, for which the oscillation period of the ideal harmonic oscillator is $2 \pi$, which approximately corresponds to the oscillation period in this simulation as well.

the box should be chosen sufficiently far away from the degeneracy point such that its ground state is a charge state. A nonvanishing gate voltage must be applied to the large island so that the corresponding gate charge $N_{G}$ is (preferably much) larger than 1. At $t=0, N_{G}$ is switched to zero, on a time scale which is short compared to the oscillation period. This will start coherent oscillations of a frequency close to $\sqrt{2 E_{J} E_{C}}$. Similarly, the box gate charge $N_{G B}$ must be switched to (or near) the value of $1 / 2$ corresponding to the degeneracy point, so that its old ground state now becomes a superposition of the two new energy eigenstates (with equal weights in the case of $N_{G B}=1 / 2$ ). The subsequent time evolution of the entire system will lead to the entangled "Schrödinger cat" -like state described above. After a time of the order of $t \approx \delta \omega / g^{2}$, i.e.,

$$
t \approx\left[\hbar E_{C}\left(1-E_{J B} / \sqrt{2 E_{J} E_{C}}\right)\right] E_{C C}^{-2},
$$

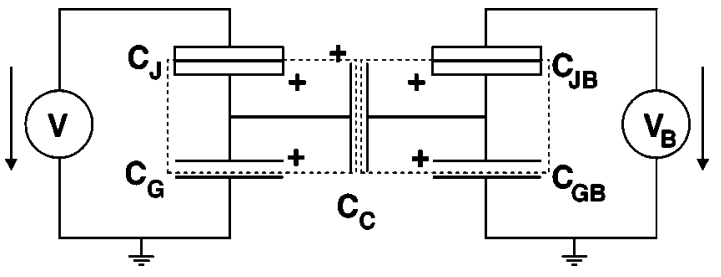

FIG. 3. Circuit diagram for the system shown in Fig. 1. The two superconducting islands appearing as boxes in Fig. 1 correspond to the regions marked by dashed rectangles. The junction and gate capacitances are $C_{J}$ and $C_{G}$ for the larger island and $C_{J B}, C_{G B}$ for the "box." The coupling capacitance is denoted by $C_{C}$ and the gate voltages are $V$ and $V_{B}$.

when the desired state has been produced, it is to be measured. To this end, it is preferable to "freeze" the charge dynamics both of the box and the oscillator. For the case of the box, this can be done by switching the box gate charge $N_{G B}$ back to zero. The Josephson coupling $E_{J}$ of the oscillator could be set to zero if the junction connecting the large island and the corresponding bulk superconductor (marked by $C_{J}$ in Fig. 3) is replaced by a dc-SQUID ring, whose effective $E_{J}$ depends on the flux threading the ring and vanishes for $\Phi=\Phi_{0} / 2$. The charges on the small and large superconducting islands can then be measured like in Nakamura's original experiment via the current that flows through the "probe leads" that have been coupled to the islands through tunnel junctions. Alternatively, one could make use of the single-electron transistor as a measurement device ${ }^{10}$ for both charge states.

The probability $P$ to measure no excess Cooper pair on the box initially oscillates between 0 and 1 , since the box starts in an equal superposition of its new energy eigenstates at the degeneracy point. After some time, the coupling to the large island gradually leads to entanglement of the box eigenstates with the coherent oscillator states. Since the frequency of the oscillation depends on the box eigenstate, the phases of the two oscillator states become distinct. Therefore, the overlap of these states becomes zero, so that the reduced density matrix of the box describes an equal mixture of the two charge states. This suppresses the observed oscillations in the probability $P$. In the ideal case (at least), one may observe a revival of these oscillations, once the accumulated phase difference reaches the value of $2 \pi$.

The experimental parameters corresponding to the numerical simulation above are the following. Taking the charging energy of the large island to be $E_{C}=10 \mu \mathrm{eV}$ (compare the thermal energy, e.g. $k_{B} T \approx 30 \mathrm{mK} \approx 3 \mu \mathrm{eV}$ ), this leads to a Josephson coupling energy of $E_{J}=50 E_{C}$ $=500 \mu \mathrm{eV}$. The latter should be smaller than the gap-this condition is not fulfilled for Al used in Nakamura's experiment. The parameters of the box are $E_{J B}=9 E_{C}=90 \mu \mathrm{eV}$ and $E_{C B}=1 \mathrm{meV}$, which is again larger than the gap. Note that the size of $E_{C B}$ does not affect the results of the simulations described above, but if it is too small-compared with $E_{J B}$ - the approximation of a two-level system is no longer justified. The charging energy connected with the capacitive coupling is $E_{C C}=0.1 E_{C}=1 \mu \mathrm{eV} \approx 10 \mathrm{mK}$. This is probably smaller than the thermal energy, but the influence 
of thermal fluctuations on the dynamics depends on the strength of the coupling to the environment and the time duration of the experiment.

\section{CONCLUSIONS}

We have analyzed the model of two capacitively coupled superconducting islands, one of which can be considered to be a two-level system and the other one an approximation to a harmonic oscillator, whose oscillation frequency depends on the state of the two-level system. Placing the former in a coherent superposition and the latter in an oscillation of the average charge number will result in a time evolution that leads to mesoscopically different states of the large superconducting island (so that its wave function is peaked at different values of the charge number). We have presented results of numerical simulations taking into account the deviations of the system from the ideal case of a two-level system coupled to a perfect harmonic oscillator. A measurement of the resulting "Schrödinger cat'" state could be realized using the techniques pioneered in the experiment of Nakamura et al.

\section{ACKNOWLEDGMENTS}

We would like to thank Mahn-Soo Choi and R. Fazio for discussions and the Swiss National Science Foundation for financial support.

\section{APPENDIX: CIRCUIT DIAGRAM AND RELATIONS BETWEEN THE PARAMETERS}

The relations connecting the charges and the voltages on the different capacitors are the following, where $Q$ is the total charge on the larger island (see Fig. 3):

$$
\begin{gathered}
Q \equiv Q_{G}+Q_{J}+Q_{C}, \\
V_{G}=V_{J}+V, \\
V_{G}=V_{C}+V_{G B} .
\end{gathered}
$$

There are two further equations that result from replacing $Q$ by $Q_{B}, V_{G}$ by $V_{G B}$, etc. in the first two of these lines. In (A1), $Q_{C}$ must be replaced by $-Q_{C}$.

The charging energy is given by

$$
\begin{aligned}
E_{c h}= & \frac{Q_{J}^{2}}{2 C_{J}}+\frac{Q_{G}^{2}}{2 C_{G}}+V\left(Q-Q_{J}\right)+\frac{Q_{C}^{2}}{2 C_{C}}+\frac{Q_{J B}^{2}}{2 C_{J B}}+\frac{Q_{G B}^{2}}{2 C_{G B}} \\
& +V_{B}\left(Q_{B}-Q_{J B}\right) .
\end{aligned}
$$

Using all of the equations given above, together with the usual relation between charge, voltage, and capacitance for each of the capacitors, the charging energy $E_{c h}$ can be rewritten as a function of the total charges $Q$ and $Q_{B}$ alone:

$$
\begin{aligned}
E_{c h}= & \frac{E_{C}}{(2 e)^{2}}(Q+\widetilde{Q})^{2}+\frac{E_{C B}}{(2 e)^{2}}\left(Q_{B}+\widetilde{Q}_{B}\right)^{2} \\
& +\frac{E_{C C}}{(2 e)^{2}} Q Q_{B}+\text { const. }
\end{aligned}
$$

Here the following abbreviations have been introduced:

$$
\begin{gathered}
E_{C C} \equiv \frac{(2 e)^{2} C_{C}}{\left(C_{G}+C_{J}+C_{C}\right)\left(C_{G B}+C_{J B}+C_{C}\right)-C_{C}^{2}}, \\
E_{C} \equiv 2 e^{2}\left(C_{G B}+C_{J B}+C_{C}\right)\left(E_{C C} / C_{C}\right), \\
\widetilde{Q} \equiv\left(C_{G B}+C_{J B}+C_{C}\right)^{-1}\left\{-V_{B} C_{J B} C_{C}+\left[C_{C} C_{G}\right.\right. \\
\left.\left.+\left(C_{G B}+C_{J B}\right)\left(C_{C}+C_{G}\right)\right] V\right\} .
\end{gathered}
$$

The quantities $E_{C B}$ and $\widetilde{Q}_{B}$ can be obtained by interchanging $C_{G}$ with $C_{G B}, V$ with $V_{B}$, etc., in the expressions for $E_{C}$ and $\widetilde{Q}$.

In the main text the charging energy was used in the form

$$
E_{C}\left(N-N_{G}\right)^{2}+E_{C B}\left(N_{B}-N_{G B}\right)^{2}+E_{C C} N\left(N_{B}-\frac{1}{2}\right),
$$

where $N \equiv Q /(2 e), N_{B} \equiv Q_{B} /(2 e)$, and the offsets $N_{G}$ and $N_{G B}$ introduced by the gates are

$$
\begin{aligned}
N_{G}= & -V \frac{C_{C} C_{G}+\left(C_{C}+C_{G}\right)\left(C_{G B}+C_{J B}\right)}{2 e\left(C_{C}+C_{G B}+C_{J B}\right)} \\
& +V_{B} \frac{C_{J B} C_{C}}{2 e\left(C_{C}+C_{G B}+C_{J B}\right)}-\frac{C_{C}}{2\left(C_{C}+C_{G B}+C_{J B}\right)} \\
N_{G B}= & -V_{B} \cdot \frac{C_{C}\left(C_{G}+C_{J}\right)+C_{G B}\left(C_{C}+C_{G}+C_{J}\right)}{2 e\left(C_{C}+C_{G}+C_{J}\right)} \\
& +V \frac{C_{J} C_{C}}{2 e\left(C_{C}+C_{G}+C_{J}\right)} .
\end{aligned}
$$

In the limit of vanishing coupling capacitance $C_{C}$, the value of $N_{G}$ tends to the usual expression, $-V C_{G} /(2 e)$ (analogously for $\left.N_{G B}\right)$. Likewise, $E_{C}$ becomes $2 e^{2}\left(C_{G}\right.$ $\left.+C_{J}\right)^{-1}$.

The values for $E_{C}, E_{C B}$, and $E_{C C}$ quoted in the text can be obtained by choosing the capacitances to be (for example) $C_{J}=2.75 \times 10^{-16} \mathrm{~F}, \quad C_{J B}=0.5 \times 10^{-16} \mathrm{~F}, \quad C_{G}=3.2$ $\times 10^{-14} \mathrm{~F}, C_{G B}=2.5 \times 10^{-16} \mathrm{~F}, C_{C}=1.6 \times 10^{-17} \mathrm{~F}$. Since there are only three equations for five unknowns, it is possible to choose other values, within a certain range. 
*Email address: Florian.Marquardt@unibas.ch

†Email address: Christoph.Bruder@unibas.ch

${ }^{1}$ Y. Nakamura, Y.A. Pashkin, and J.S. Tsai, Nature (London) 398, 786 (1999).

${ }^{2}$ A. Shnirman, G. Schön, and Z. Hermon, Phys. Rev. Lett. 79, 2371 (1997).

${ }^{3}$ D.V. Averin, Solid State Commun. 105, 659 (1998).

${ }^{4}$ More precisely, the phrase "Josephson coupling energy of an island" refers to the coupling between the island and the adjacent bulk superconductor.

${ }^{5}$ M. Brune et al., Phys. Rev. Lett. 77, 4887 (1996).

${ }^{6}$ C.C. Gerry, Phys. Rev. B 57, 7474 (1998).

${ }^{7}$ J.E. Mooij et al., Science 285, 1036 (1999).

${ }^{8}$ A.A. Odintsov and A. Vourdas, Europhys. Lett. 34, 385 (1996).

${ }^{9}$ O. Buisson and F.W.J. Hekking, cond-mat/0008275 (2000).

${ }^{10}$ A. Shnirman and G. Schön, Phys. Rev. B 57, 15400 (1998). 